\title{
BK polyomavirus infection following COVID-19 infection in renal transplant recipients: a single-center experience
}

\author{
Hari Shankar Meshram, Vivek B. Kute, Sanshriti Chauhan \\ Department of Nephrology and Clinical Transplantation, Institute of Kidney Diseases and Research Center, Dr. HL Trivedi Institute of \\ Transplantation Sciences (IKDRC-ITS), Ahmedabad, India
}

Coronavirus disease 2019 (COVID-19) has affected the transplantation community worldwide. Reports of transplant patients acquiring COVID-19 infections are extensive with diverse mortality rates [1]. Follow-up studies of COVID-19 in transplant communities are lacking. There are limited data on the association of the BK polyomavirus (BKPyV) with active COVID-19 infection in kidney transplant recipients (KTRs) [2,3]. Currently, theoretical concerns exist related to graft dysfunction or loss during the post-COVID-19 follow-up period in KTRs. This study aimed to explore the clinical profile, outcomes, and follow-up experiences of KTR patients who developed BKPyV after COVID-19. This was a single-center retrospective analysis of a study approved by our Institutional Ethical Board (ECR/143/Inst/GJ/2013/RR19 with application No: EC/App/20Jan21/08) and was conducted in compliance with the Declaration of Helsinki. KTR patients admitted for COVID-19 infection during the study period from June 2020 to December 2020 who developed BKPyV after a positive COVID-19 diagnosis were included. We conducted extended and close monitoring and follow-up of the cohort in the physical, clinical, and psychological domains. Follow-up BKPyV testing was conducted at 1-month after discharge, followed by every 3 months thereafter. Testing also was performed in cases of increasing creatinine.

We identified 11 cases of BKPyV after infection in 167 total COVID-19 KTR cases. Table 1 shows the overall summary of the study. The median age of the cohort was 45 years (range, 29-56 years), with male predominance (90.9\%). The majority of the cohort had comorbidities $(72.7 \%)$, underwent live-related-donor transplantation (72.7\%), and received thymoglobulin (81.8\%) upon admission for COVID-19. The baseline median serum creatinine was $1.44 \mathrm{mg} / \mathrm{dL}$ (range, $1.3-1.9 \mathrm{mg} / \mathrm{dL}$ ). COVID-19 severity was categorized as mild (9\%), moderate (45\%), and severe cases (46\%) [4]. Acute kidney injury was reported in all cases, and acute respiratory distress syndrome developed in $18.2 \%$ of KTR patients, with one fatality during COVID-19 admission. Five cases (45.5\%) received steroids during acute COVID-19 infection. At baseline, no cases showed BKPyV in the blood. Baseline polymerase chain reaction (PCR) urine testing of the cohort

Received: April 13, 2021; Revised: May 16, 2021; Accepted: May 23, 2021

Editor: Seung-Yeup Han, Keimyung University, Daegu, Republic of Korea

Correspondence: Hari Shankar Meshram

Department of Nephrology and Clinical Transplantation, Institute of Kidney Diseases and Research Center, Dr. HL Trivedi Institute of

Transplantation Sciences (IKDRC-ITS), Civil Hospital Campus, Asarwa, Ahmedabad, Gujarat 380016, India.

E-mail: harishankarmania@gmail.com,hsnephrology@gmail.com

ORCID: https://orcid.org/0000-0001-9148-8168

Copyright (C) 2021 by The Korean Society of Nephrology

(a) This is an Open Access article distributed under the terms of the Creative Commons Attribution Non-Commercial and No Derivatives License (http:// creativecommons.org/licenses/by-nc-nd/4.0/) which permits unrestricted non-commercial use, distribution of the material without any modifications, and reproduction in any medium, provided the original works properly cited. 


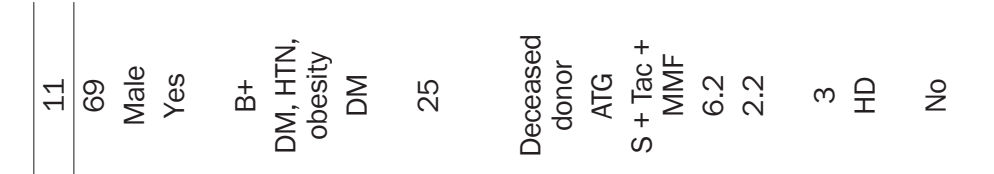

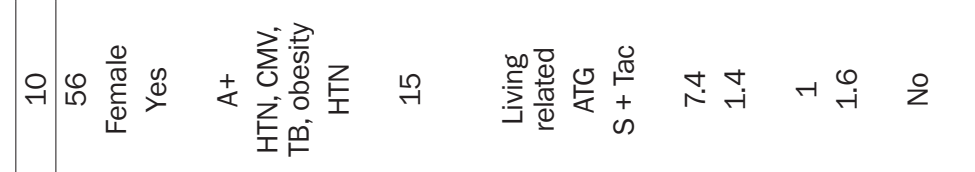

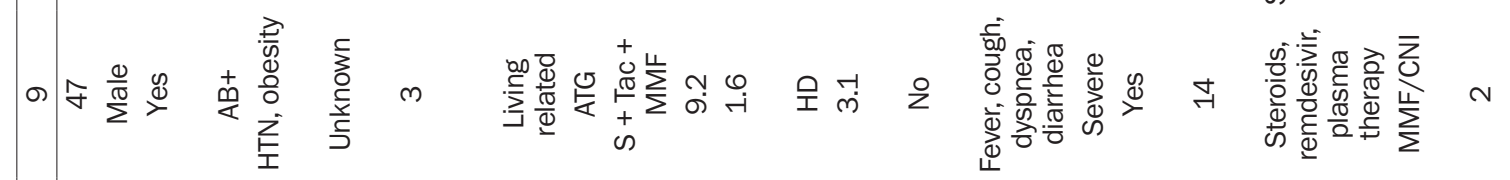

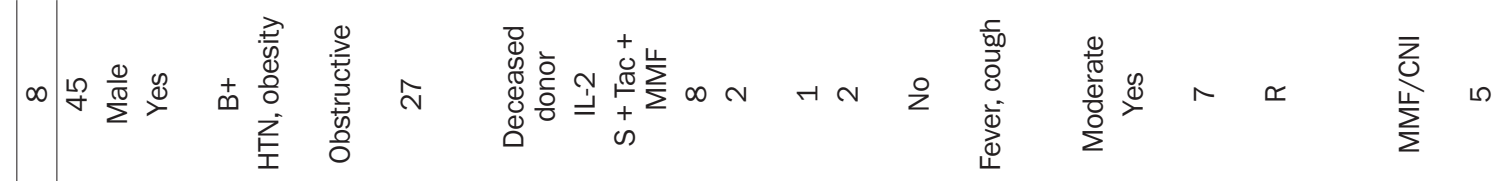

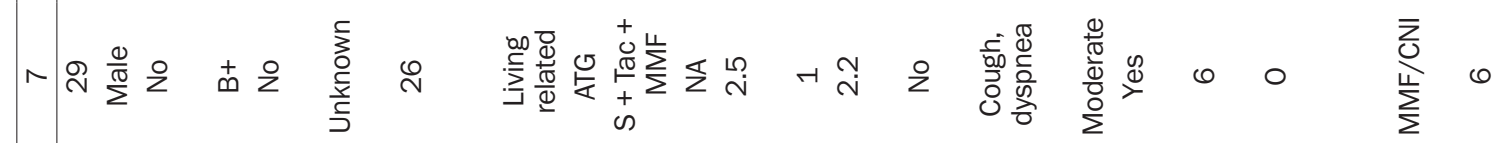

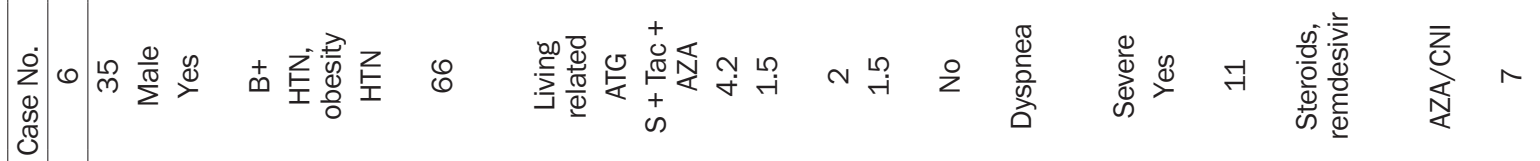

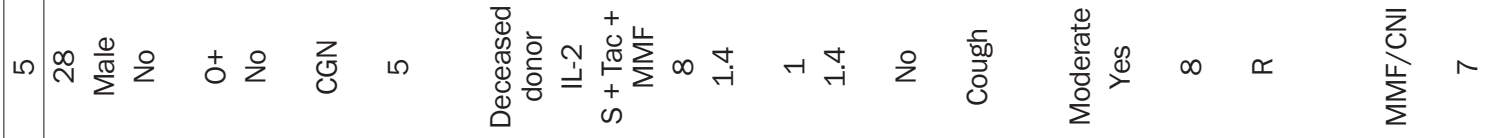

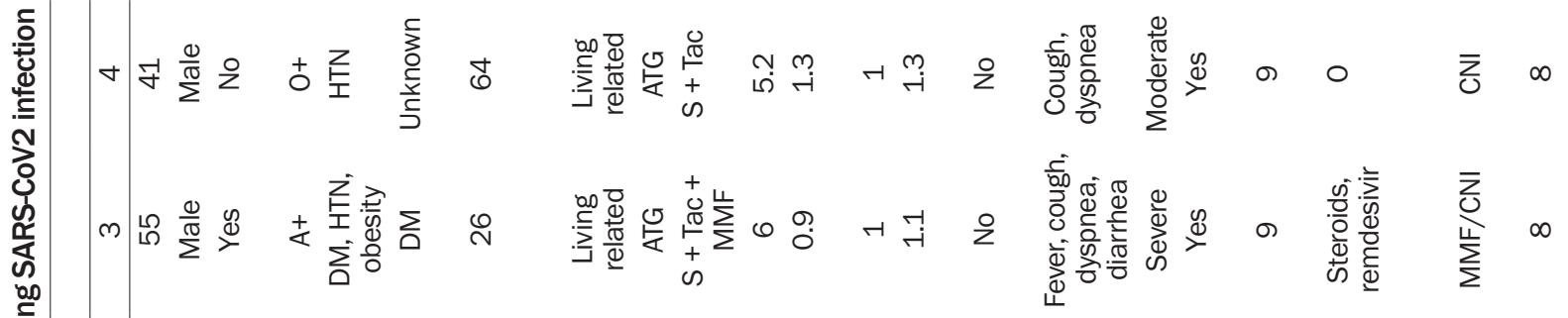

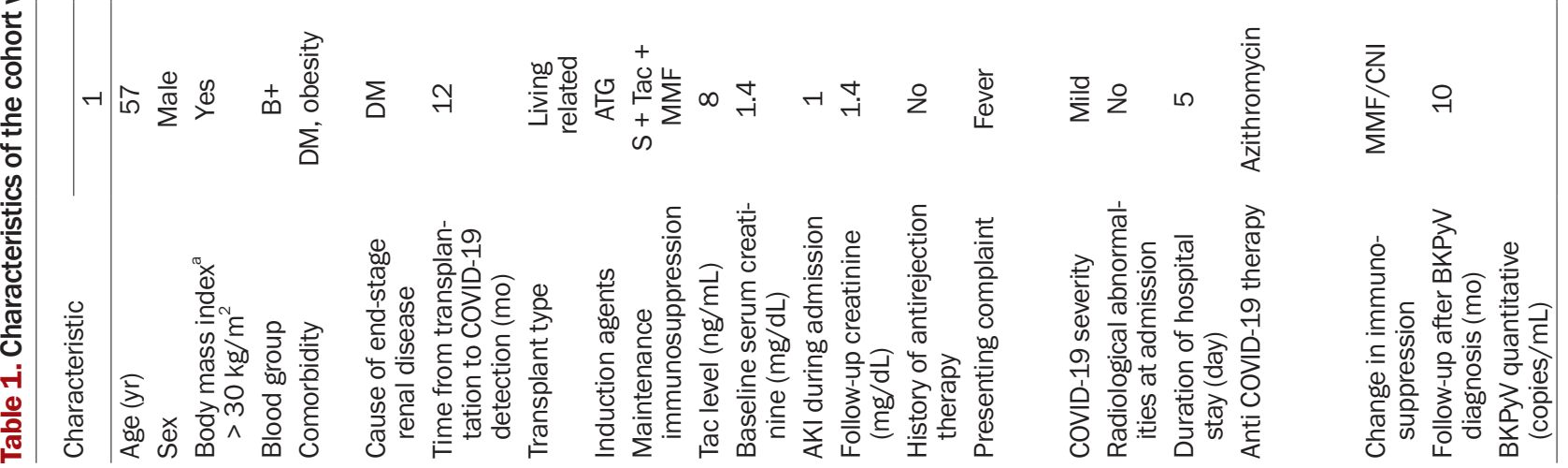




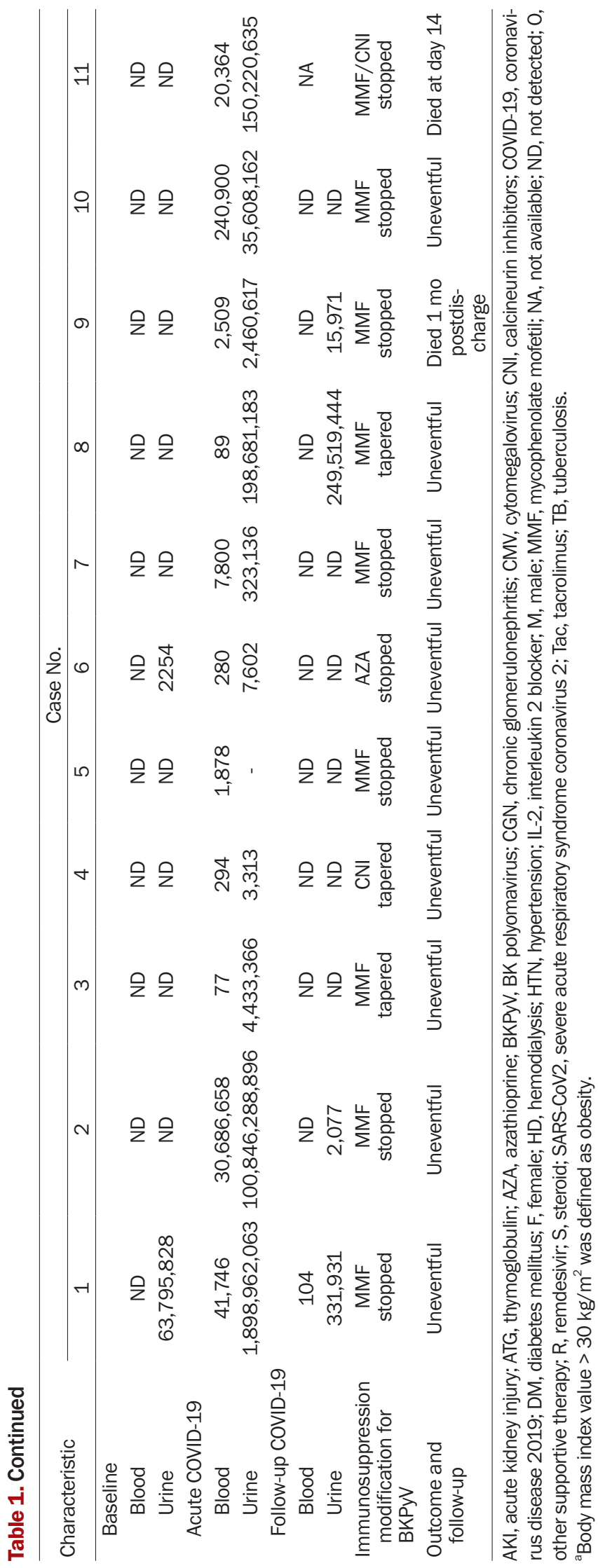

did not detect BKPyV in most cases (81.8\%). Table 2 shows the laboratory parameters of the cohort. The median BKPyV blood and urine PCR results during acute COVID-19 infection were 2,509 copies/mL (range, 280-41,746 copies/mL) and 4,433,366 copies/mL (range, 7,602- 198,681,183 copies/ $\mathrm{mL}$ ), respectively.

The follow-up period after BKPyV diagnosis was 7 months (range, 5-8 months). BKPyV was detected in the blood during the follow-up period in only one patient. The BKPyV PCR urine values of the cohort were less than those detected in $63.6 \%$ of the follow-up cases. No graft loss or graft dysfunction was reported in the cohort. No patient developed sensitization, urine microhematuria, or proteinuria during the follow-up period. Radiological resolution [5] of COVID-19 infection was defined as the absence of any chest radiographic abnormality potentially related to the infection; this type of resolution was seen in $91.6 \%$ of KTR cases and resolved after a median of 3 months of follow-up. No multisystemic sequelae were reported. One case was readmitted 1 week after discharge and died due to secondary fungal infection (aspergillosis) after 1 month.

Our report could simply indicate that the natural history and course of BKPyV happened to coincide with COVID-19 infection, and there might be no actual association between the two; however, reactivation of viruses like BKPyV is a high-risk factor for graft loss in transplant patients [6]. BKPyV causes complex changes in immunity and weakens the immune response, which could potentially aggravate the immune/graft injury often present in COVID-19 infection [7]. Elevated levels of inflammatory cytokines in COVID-19 infection can lead to greater transcription of the BKPyV genome [8]. The use of thymoglobulin as an induction agent could have been a confounding factor for BKPyV, but the institutional protocol of using a low dosage of thymoglobulin $(1.5 \mathrm{mg} / \mathrm{kg}$ ) hinders this connection. Moreover, at our center, the incidence of BKPyV in COVID-19 patients was 6.6\% (11 of 167 patients), which was higher than the rate reported in normal follow-up or in non-COVID-19 admissions (1.3\%). While we were unable to show a definite association of BKPyV with COVID-19 infection, the use of steroids to treat these patients and COVID-19 infection itself are both risk factors for an increase in number of BKPyV in KTRs. Therefore, we suggest screening for BKPyV in COVID-19 patients.

One limitation of this study was its small sample size. To date, this is the largest cohort of KTRs with BKPyV after 
Table 2. Laboratory and inflammatory markers in the cohort at admission due to COVID-19 infectio

\begin{tabular}{|c|c|c|c|c|c|c|c|c|c|c|c|}
\hline \multirow{2}{*}{ Laboratory parameter (normal range) } & \multicolumn{11}{|c|}{ Case No. } \\
\hline & 1 & 2 & 3 & 4 & 5 & 6 & 7 & 8 & 9 & 10 & 11 \\
\hline Hemoglobin (13-16 g/dL) & 10.6 & 12.5 & 13.1 & 11.4 & 14.8 & 14.4 & 17.1 & 9.3 & 17.1 & 8.5 & 7.8 \\
\hline Total leukocyte count $\left(4-11 \times 10^{3}\right.$ cells $\left./ \mathrm{L}\right)$ & 3,280 & 4,990 & 2,660 & 5,370 & 5,040 & 12,400 & 6,800 & 4,850 & 11,830 & 4,000 & 13,600 \\
\hline Polymorphs $(60 \%-70 \%)$ & 71 & 75 & 55 & 72 & 62 & 78 & 90 & 74 & 8 & 69 & 83 \\
\hline Lymphocyte (25\%-33\%) & 26 & 23 & 42 & 25 & 35 & 19 & 8 & 23 & 16 & 27 & 15 \\
\hline Platelet counts $\left(150-400 \times 10^{9}\right.$ cells/L) & 441 & 190 & 272 & 178 & 244 & 261 & 124 & 282 & 285 & 178 & 279 \\
\hline Procalcitonin (<0.5 ng/mL) & 0.05 & 0.05 & 0.05 & NA & 0.05 & 0.27 & 0.06 & 0.31 & 11.6 & 7.9 & NA \\
\hline Highly sensitive $\mathrm{C}$ protein (0-10 mg/L) & 29 & 30.9 & 4.8 & 6.1 & 21.8 & 51.4 & 42.1 & 74.2 & 210 & NA & 189 \\
\hline Aspartate transferase $(0-40 \mathrm{IU} / \mathrm{L})$ & 59 & 42 & 30 & 18 & 24 & 32 & 19 & 25 & 18 & 40 & 11 \\
\hline Interleukin $6(<7 \mathrm{pg} / \mathrm{mL})$ & 8.2 & 58.3 & NA & 1,531 & NA & NA & 25.7 & 14.5 & 24.1 & NA & 470 \\
\hline Lactate dehydrogenase (100-190 IU/L) & 387 & 510 & 272 & 322 & 391 & 523 & 550 & 292 & NA & NA & 534 \\
\hline
\end{tabular}

COVID-19, coronavirus disease 2019; NA, not available.

\section{COVID-19 infection.}

In summary, we report BKPyV following COVID-19 with no graft loss during the follow-up period. We suggest screening for BKPyV in all renal transplant patients with active COVID-19 infection (especially in patients with a history of $\mathrm{BKPyV}$ and in severe COVID-19 infection) as a safe option to avoid complications.

\section{Conflicts of interest}

All authors have no conflicts of interest to declare.

\section{Authors' contributions}

Conceptualization, Data curation, Formal analysis, Investigation: All authors

Writing-original draft: All authors

Writing-review \& editing: All authors

All authors read and approved the final manuscript.

\section{ORCID}

Hari Shankar Meshram, https://orcid.org/0000-0001-9148-8168 Vivek B. Kute, https://orcid.org/0000-0002-0002-2854

Sanshriti Chauhan, https://orcid.org/0000-0001-7385-5614

\section{References}

1. Raja MA, Mendoza MA, Villavicencio A, et al. COVID-19 in solid organ transplant recipients: a systematic review and meta-analysis of current literature. Transplant Rev (Orlando) 2021;35:100588.

2. Masset C, Ville S, Halary F, et al. Resurgence of BK virus following Covid-19 in kidney transplant recipients. Transpl Infect Dis 2021;23:e13465.

3. Virmani S, Gleeson SE, Girone GF, et al. Identifying a kidney transplant recipient COVID phenotype to aid test utilization in the setting of limited testing availability-does one exist? Transplant Proc 2020;52:2584-2591.

4. Directorate General of Health Services (EMR Division), Ministry of Health \& Family Welfare, Government of India. Clinical management protocol: COVID-19. Version 3 [Internet]. New Delhi: Ministry of Health \& Family Welfare, Government of India; 2020 June 13 [cited 2021 Jul 27]. Available from: https://www.mohfw. gov.in/pdf/ClinicalManagementProtocolforCOVID19.pdf.

5. Bruns AH, Oosterheert JJ, El Moussaoui R, Opmeer BC, Hoepelman AI, Prins JM. Pneumonia recovery: discrepancies in perspectives of the radiologist, physician and patient. J Gen Intern Med 2010;25:203-206.

6. Blazquez-Navarro A, Dang-Heine C, Wittenbrink N, et al. BKV, CMV, and EBV interactions and their effect on graft function one year post-renal transplantation: results from a large multi-centre 
study. EBioMedicine 2018;34:113-121.

7. Chen Y, Trofe J, Gordon J, et al. Interplay of cellular and humoral immune responses against BK virus in kidney transplant recipients with polyomavirus nephropathy. J Virol 2006;80:3495-3505.
8. Bethge T, Hachemi HA, Manzetti J, Gosert R, Schaffner W, Hirsch HH. Sp1 sites in the noncoding control region of BK polyomavirus are key regulators of bidirectional viral early and late gene expression. J Virol 2015;89:3396-3411. 